Pacific Journal of Mathematics

ON THE GEOMETRY OF THE UNIT BALL IN THE SPACE OF 


\title{
ON THE GEOMETRY OF THE UNIT BALL IN THE SPACE OF REAL ANNIHILATING MEASURES
}

\author{
P. R. AHERN
}

Our purpose is to study the geometry of the unit ball in the space of real measures on the boundary of a finite Riemann surface that annihilate the analytic functions on that surface with continuous boundary values. We show that the number of boundary components of the surface (and hence the topological type) can be determined from the geometry of this unit ball. More precisely, if the surface has $k$ boundary components then this unit ball has $2^{k}-2$ "flat faces" of highest possible dimension. We also get some information on extreme points and conclude with an example to show that the linear structure of this unit ball depends not only on the topology of the surface but also on some of its conformal structure.

Let $R$ be a finite Riemann surface with boundary $\Gamma$, and let $A$ denote the algebra of complex valued functions that are continuous on $\bar{R}=R \cup \Gamma$ and holomorphic on $R$, and let $\operatorname{Re} A$ denote the space of real parts of functions in $A$. If $n$ is the first Betti number of $R$ then it is well-known that $\operatorname{Re} A$ has codimension $n$ in $C_{R}(\Gamma)$, the space of continuous real valued functions on $\Gamma$. In other words, the space $N$ of real measures on $\Gamma$ that annihilate $A$ has dimension $n$ over the real numbers.

Fix $\xi \in R$ and let $d m$ denote the unique positive measure on $\Gamma$ such that

$$
u(\xi)=\int u d m
$$

for all $u$ that are continuous on $\bar{R}$ and harmonic on $R$. We know the following, from [4]: if $d \nu \in N$ then $d \nu=h d m$ where $h$ is the restriction to $\Gamma$ of a function that is meromorphic on $\bar{R}$ and regular on $\Gamma$. Moreover there is a meromorphic differential $\omega$ on $\bar{R}$ that is regular and nonvanishing on $\Gamma$ and real along $\Gamma, \omega$ has the property that if $h d m \in N, h \omega$ is holomorphic on $\bar{R}$. Also " $\omega=d m$ " in the sense that

$$
\int u d m=\int_{\Gamma} u \omega
$$

for every continuous function $u$ on $\Gamma$. Hence if $h$ is meromorphic on $\bar{R}$ and real along $\Gamma$ then $h d m \in N$ because for all $f \in A$

$$
\int f h d m=\int_{\Gamma} f h \omega=0
$$


since $h \omega$ is a holomorphic differential. Finally we know that $\omega$ has a simple pole at $\xi$, is otherwise regular on $\bar{R}$, and vanishes precisely at the $n$ critical points of the Greens function for $R$ with pole at $\xi$. If $\theta$ is the divisor of $\omega$ we have:

Lemma 1. If $h$ is a real valued function in $L^{1}(d m)$ then $h d m \in N$ if and only if $h$ is meromorphic on $\bar{R}$ and a multiple of $\theta^{-1}$ on $R$.

So we have now identified $N$ with

$N_{1}=\left\{h \in L^{1}(d m): h\right.$ is real valued on $\Gamma$, meromorphic on $\bar{R}$ and a multiple of $\theta^{-1}$ on $R$ \}. Note that if $d \nu=h d m \in N$ then the total variation of $d \nu$ is just $\int|h| d m$. Note also that no nonzero element of $N_{1}$ can vanish on a set of positive measure, $d m$.

Let $D$ denote the space of holomorphic differentials on $\bar{R}$ that are real along $\Gamma$, then $D$ has dimension $n$ over the real numbers. If $h \in N$, then $h \omega \in D$; since the correspondence $h \rightarrow h \omega$ is one to one and $N_{1}$ and $D$ have the same dimension we see that this correspondence maps $N_{1}$ isomorphically onto $D$. Since $\omega$ doesn't vanish on $\Gamma, h \omega$ vanishes on $\Gamma$ if and only if $h$ vanishes on $\Gamma$.

Before discussing the geometry of the unit ball of $N$, we need some elementary geometrical facts. Let $N$ be an $n$-dimensional vector space over the real numbers with a norm \|\| . Let

$$
\begin{aligned}
& B=\{x \in N:\|x\| \leqq 1\} \\
& S=\{x \in N:\|x\|=1\} .
\end{aligned}
$$

A hyperplane is a translate of an $(n-1)$ dimension subspace. A hyperplane $H$ is said to be a supporting hyperplane if $H \cap S=H \cap B \neq \varnothing$. If $H$ is a supporting hyperplane and $H \cap S$ has nonempty interior relative to $H$ then that interior is called a face of $S$. Note that if $H$ is a hyperplane and $H \cap S$ has nonempty interior relative to $H$, then $H$ is a supporting hyperplane. Note also that if $x$ belongs to a face then there is a unique supporting hyperplane containing $x$. If $x, y \in N$ we let $[x, y]$ and $(x, y)$ be the closed and open segments joining $x$ and $y$. We omit the proofs of the following two elementary lemmas.

Lemma 2. If $F_{1}$ and $F_{2}$ are faces of $S$ and $x_{1} \in F_{1}, x_{2} \in F_{2}$ then $F_{1}=F_{2}$ if and only if $\left[x_{1}, x_{2}\right] \subseteq S$.

Lemma 3. If $x, x_{2}, \cdots, x_{n} \in S$ and are linearly independent and $y_{2}, \cdots, y_{n} \in S\left[x_{i}, y_{i}\right] \subseteq S$ for $i=2, \cdots, n$ and $x \in\left(x_{i}, y_{i}\right) \quad i=2, \cdots, n$; then $x$ lies on a face of $S$.

Now return to $N_{1}$, recalling that no nonzero element of $N_{1}$ can 
vanish on a set of positive measure, $d m$, we can now apply a result of de Leeuw and Rudin. We include a proof since our statement is slightly different.

LEMma 4. (de Leeuw-Rudin [2]) Let $h, g \in S$ be linearly independent, if $\pi(h, g)$ denotes the plane through the origin containing $h$ and $g$, then $\pi(h, g) \cap S$ contains a line segment one of whose interior points is $h$ if and only if $g / h$ is bounded on $\Gamma$.

Proof. Suppose $\varphi=g / h$ is bounded, let $\alpha=\int \varphi|h| d m$ and then choose $\varepsilon$ so small that $-1 \leqq \varepsilon(\varphi-\alpha) \leqq 1$, then

$$
\begin{aligned}
\int \mid h & \pm \varepsilon(\varphi-\alpha) h \mid d m \\
& =\int|1 \pm \varepsilon(\varphi-\alpha)||h| d m \\
& =\int(1 \pm \varepsilon(\varphi-\alpha))|h| d m=1 .
\end{aligned}
$$

Now $h \pm \varepsilon(\varphi-\alpha) h=h \pm \varepsilon g \mp \varepsilon \alpha h \in \pi(g, h)$ and $h$ is the midpoint of the line joining $h+\varepsilon(\varphi-\alpha) h$ to $h-\varepsilon(\varphi-\alpha) h$. On the other hand, suppose $f_{1}, f_{2} \in S \cap \pi(g, h)$ and $h=1 / 2\left(f_{1}+f_{2}\right)$ this means there is

$$
0 \neq f \in \pi(g, h) \int|h+f| d m=1
$$

so

$$
\int|1 \pm f / h \| h| d m=1
$$

iso

$$
-1 \leqq f / h \leqq 1 \quad \text { a.e. } d m
$$

and $\int f / h|h| d m=0$, i.e., $f / h$ not constant. Now $f=\alpha g / h+\beta$, since $f / h$ is not constant $\alpha$ is not zero and hence $g / h$ is bounded.

Now suppose $h \in S$, and $h$ doesn't vanish on $\Gamma$ and $g \in S$ with $g$, $h$ linearly independent then $g / h$ is bounded on $\Gamma$ so, as in the proof of the previous lemma, there is an $\alpha$ such that for all sufficiently small $|\varepsilon|,(1-\varepsilon \alpha) h+\varepsilon g \in S$. Now let $h, g_{2} \cdots, g_{n}$ be a basis for $N$. By the same argument there are $\alpha_{2}, \cdots, \alpha_{n}$ so that $\left(1-\varepsilon \alpha_{i}\right) h+\varepsilon g_{i} \in S$ for all sufficiently small $|\varepsilon|, i=2, \cdots, n$. Fix such a small $\varepsilon>0$ and consider the functions $f_{i}=\left(1-\varepsilon \alpha_{i}\right) h+\varepsilon g_{i}, i=2, \cdots, n$. First $h$, $f_{2} \cdots, f_{n}$ are linearly independent because the matrix that expresses these elements in terms of the basis $h, g_{2}, \cdots, g_{n}$ has determinant $\varepsilon^{n-1}$. By Lemma $3, h$ lies on a face of $S$. On the other hand suppose $h$ lies on a face $F$ of $S$. If $g \in F, g \neq h$ then clearly $\pi(g, h) \cap S$ contains 
a line segment with $h$ as an interior point and so $g / h$ is bounded on $\Gamma$. Choose $g_{2}, \cdots, g_{n} \in F$ so that $h, g_{2}, \cdots, g_{n}$ are linearly independent. Arguing as above we see that $g_{i} / h$ is bounded on $\Gamma$ for $i=2, \cdots, n$ and it follows that $g / h$ is bounded on $\Gamma$ for all $g \in N$. Suppose there were $\eta \in \Gamma$ such that $h(\eta)=0$, then it would follow that $g(\eta)=0$ for all $g \in N$. Multiplying by $\omega$ we see that this would imply that $\tau(\eta)=0$ for all $\tau \in D$. If $\varphi$ is a holomorphic differential on the doubled surface $\hat{R}$ of $R$, then $\varphi=\tau_{1}+i \tau_{2}$ on $R$ where $\tau_{1}, \tau_{2} \in D_{1}$ [1], so we would have that every holomorphic differential on $\hat{R}$ vanishes at $\eta$, that this is not the case follows from the Riemann-Roch theorem for instance. Now let $h, g \in S$; neither of which vanishes on $\Gamma$ then each lies on a face, and they lie on the same face if and only if $[h, g] \subseteq S$, i.e.,

$$
\int|t h+(1-t) g| d m=1
$$

for $0 \leqq t \leqq 1$, but

$$
\int|t h+(1-t) g| d m \leqq t \int|h| d m+(1-t) \int|g| d m=1
$$

and equality holds if and only if $h$ and $g$ have the same sign on $\Gamma$.

Now we have the following theorem.

THEOREM 1. Let $h$ be in the unit sphere $S$ of $N_{1}$, then $h$ lies on a face if and only if $h$ doesn't vanish on $\Gamma$. Moreover, if $h, g \in S$, neither vanishes on $\Gamma$, then $h$ and $g$ lie on the same face if and only if $h$ and $g$ have the same sign on $\Gamma$.

Now suppose $\Gamma$ has $k$ components $\Gamma_{1}, \cdots, \Gamma_{k}$. Let $1 \leqq s \leqq k-1$, and pick $s$ of these components $\Gamma_{i_{1}}, \cdots, \Gamma_{i_{s}}$. We want to show that there exists $h \in S$ so that $h>0$ on $\Gamma_{i_{1}} \cup \cdots \cup \Gamma_{i_{s}}$ and

$$
h<0 \Gamma-\left(\Gamma_{i_{1}} \cup \cdots \cup \Gamma_{i_{s}}\right) \text {. }
$$

Let $u$ be harmonic on $\bar{R}$ and $u \equiv 0$ on $\Gamma_{i_{1}} \cup \cdots \cup \Gamma_{i_{s}}$ and $u \equiv 1$ on $\Gamma-\left(\Gamma_{i_{1}} \cup \cdots \cup \Gamma_{i_{s}}\right)$. We consider the differential $\tau=-{ }^{*} d u+i d u$; in local coordinates $\tau$ takes the form

$$
\left(\frac{\partial u}{\partial y}+i \frac{\partial u}{\partial x}\right)(d x+i d y)
$$

and is therefore holomorphic. Fix a boundary component $\Gamma_{j}$ and consider a conformal map of an annulus

$$
\left\{z=r e^{i \theta}: r_{0} \leqq r \leqq 1\right\}
$$


into $\bar{R}$ so that $\{|z|=1\}$ is carried onto $\Gamma_{j}$. Then in these coordinates along $\Gamma_{j}, \tau$ has the form $-(\partial u / \partial r) d \theta$. Note that if $\Gamma_{j} \leqq \Gamma_{i_{1}} \cup \cdots \cup \Gamma_{i_{s}}$ then $-\partial u / \partial r>0$ and otherwise $-\partial u / \partial r<0$, [6] page 15. Now there exists $h \in N_{1}$ such that $h \omega \equiv \tau$ and since $\tau$ doesn't vanish on $\Gamma$ neither does $h$. Now $\int_{\Gamma_{j}} h \omega=\int_{\Gamma_{j}} \tau=-\int_{0}^{2 \pi}(\partial u / \partial r) d \theta$. So $\int_{\Gamma_{j}} h \omega>0$ if $\Gamma_{j} \subseteq \Gamma_{i_{1}} \cup \cdots \cup \Gamma_{i_{s}}$ and $^{j} \int_{\Gamma_{j}} h \omega \stackrel{\Gamma_{j}}{<} 0$ otherwise. Since $\int_{r_{j}} h \omega^{\Gamma_{j}}=\int_{\Gamma_{j}} h d m$ and $h$ must have constant sign on each $\Gamma_{j}$ it follows that $h>^{\Gamma_{j}} 0$ on $\Gamma_{i_{1}} \cup \cdots \cup \Gamma_{i_{s}}$ and $h<0$ on $\Gamma\left(\Gamma_{i_{1}} \cup \cdots \cup \Gamma_{i_{s}}\right)$. The components $\Gamma_{i_{1}}$, $\cdots, \Gamma_{i_{s}}$ can be chosen in $2^{k}-2$ different ways. Combining this with the fact two elements of $S$ that don't vanish on $\Gamma$ lie on the same face if and only if they have the same sign on $\Gamma$ we have:

THeOREM 2. If $\Gamma$ has $k$ components then $S$ has $2^{k}-2$ faces.

Next we consider the extreme points of $B$. From the de LeeuwRudin lemma we see that $h \in S$ is extreme if and only if for every $g \in N_{1}$, the meromorphic function $g / h$ is either constant or unbounded on $\Gamma$, this says that $h$ must have a "maximal number at zeros on $\Gamma$ ".

LEMMA 5. If $h \in S$, then $h$ has at most $2 n-2$ zeros on $\Gamma$, if $h$ is extreme then it has at least $n-2$ zeros on $\Gamma$. If $h$ has $2 n-2$ zeros on $\Gamma$ then $h$ is extreme.

Proof. Since $h \omega$ is holomorphic on $R, h$ has at most $n$ poles on $R$, and since $h$ is real on $\Gamma$ it can be extended by reflection to the doubled surface $\hat{R}$. On $\hat{R}, h$ has the same number of zeros as poles so if $Z$ is the number of zeros of $h$ on $\Gamma$ we have

$$
\begin{gathered}
2 Z-2 P+k=0 \quad \text { or } \\
k=2 P-2 Z \leqq 2 n-2
\end{gathered}
$$

since $P \leqq n$ and we know that $h$ vanishes at $\xi \in R$.

Now suppose that $h$ is extreme, and that $Q$ is a function that has a pole at $\xi$ and zeros at the zeros of $\omega$, then $h Q$ is an extreme point of the unit ball of $H^{1}$, again by the result of the Leeuw-Rudin. So we have [3],

$$
Z-P+n-1 \leqq n / 2
$$

Since $Z-P=-\frac{1}{2} k$ we get

$$
k \geqq n-2 \text {. }
$$

Let us assume now that $h$ has $2 n-2$ zeros on $\Gamma$. Since $k=$ $2 P-2 Z$ and $P \leqq n$ and $Z \geqq 1$, it follows that $P=n$ and $Z=1$ and 
since $h \omega$ is holomorphic and $\omega$ has exactly $n$ zeros and one pole $h \omega$ is nonvanishing. Now if $g \in N_{1}$ and $g / h$ is bounded on $\Gamma$ then $g$ has $2 n-2$ zeros on $\Gamma$ also, so $g \omega$ is nonvanishing. Therefore, $g / h=$ $g \omega / h \omega$ is holomorphic on $\bar{R}$ and real on $\Gamma$ and hence is constant. So it follows from the de Leeuw-Rudin lemma that $h$ is extreme. This finishes the proof.

If we take an $h \in S$ that is not extreme then as we have seen this means that there is a $g \in N_{1}$ such that $g$ is not a constant multiple of $h$ and $g$ vanishes at each point of $\Gamma$ where $h$ does. If $\eta$ is a point of $\Gamma$ where $g(\eta) \neq 0$ then

$$
h-\frac{h(\eta)}{g(\eta)} g \text { is an element of } N_{1}
$$

that vanishes at $\eta$ as well as all those points of $\Gamma$ that $h$ vanishes. Of course, there are infinitely many such $\eta$ so we have the following.

Lemma 6. If $h \in S$ is not extreme and $h$ has exactly $k$ zeros on $\Gamma$, then there are infinitely many distinct elements of $S$ that have at least $k+2$ zeros on $\Gamma$, the zeros of $h$ among them.

Recalling that any element of $h$ with $2 n-2$ zeros on $\Gamma$ is extreme we obtain:

CoRollary. $B$ has infinitely many extreme points.

ExAMPLES. In [3], it was seen that if $D$ is a domain in the Riemann sphere obtained by removing $n+1$ slits on the real axis then $d z / F, z d z / F, \cdots, z^{n-1} d z / F$ gives a basis for the holomorphic differentials on $D$ that are real along the boundary of $D$. Here $F(z)^{2}=$ $\left(z-e_{1}\right) \cdots\left(z-e_{2 n+2}\right)$ where $e_{1}, \cdots, e_{2 n+2}$ are the end points of the slits. Also $\tau=d z / F$ is nonvanishing on the boundary of $D$. Suppose $R$ is conformally equivalent to such a domain $D$ by means of a map $\varphi$. By means of the map $\phi$ we can bring back the differentials $\tau, z \tau, \cdots, z^{n-1} \tau$ to get a basis for the holomorphic differentials on $R$, real along $\Gamma$. This basis takes the form $\gamma, \varphi \gamma, \cdots, \varphi^{-1} \gamma$; where $\gamma$ doesn't vanish on $\Gamma$. So any holomorphic differential on $R$ that is real along $\Gamma$ takes the form $\left(\sum_{k=0}^{n-1} \alpha_{k} \varphi^{k}\right) \gamma$, where the $\alpha_{k}$ are real. Since $\varphi$ is real on $\Gamma$ it extends to give a two to one mapping of $\hat{R}$ onto the sphere. Since every real value assumed on $\hat{R}-\Gamma$ is assumed twice on $\hat{R}-\Gamma$ it follows that $\varphi$ is two to one on $\Gamma$. Take $l \leqq n-1$, pick $r_{i}$ on one of the slits $i=1, \cdots, l$, not necessarily distinct. Then $\prod_{l=1}^{l}\left(\phi-r_{i}\right) \gamma$ has exactly $2 l$ zeros on $\Gamma$, counting multiplicity. We see also that this differential is extreme if and only if $2 l=2 n-2$. 
Let $R$ be a finite Riemann surface with first Betti number $n$. Suppose there is a holomorphic differential $\gamma$ on $\bar{R}$ that is real along $\Gamma$ and that $\varphi$ has $2 n-4$ zeros on $\Gamma$ and that $\varphi$ is not extreme. Then there is another such differential $\psi$ such that $F=\psi / \varphi$ is holomorphic on $\Gamma$, has a single pole on $R$ and is real on $\Gamma$. So $F$ extends to a two to one map of $\hat{R}$ onto the Riemann sphere. Since $F$ is real along $\Gamma$ it follows, [3], that $F$ is one to one on $R$, i.e, $F$ gives a conformal map of $R$ onto a domain on the sphere whose boundary is a finite number of slits on the real axis.

Consider the case $n=3$, there are only two topological types possible: a plane domain with four boundary components or a torus with 2 discs removed. In the first case $S$ has fourteen faces, in the second it has two faces. In the planar case if the domain is equivalent to the complement of a finite number of slits on the real axis then as we have seen there are many elements in $S$ that have two zeros and are not extreme. Neither can such a point be on a face. It follows that such a point lies on a line segment that doesn't lie on any face. On the other hand if our domain is not equivalent to such a slit domain then every element of $S$ is either extreme or lies on a face. The reason is that any element of $S$ has either 0,2 , or 4 zeros on $\Gamma$. If it has two or four zeros it is extreme and if it has none it lies on a face.

\section{REFERENCES}

1. L. V. Ahlfors, Open Riemann surfaces and extremal problems on compact subregions, Comm. Math. Helv. 24 (1950), 100-134.

2. K. de Leeuw and W. Rudin, Extreme points and extremum problems in $H^{1}$, Pacific J. Math. 8 (1958).

3. T. W. Gamelin and M. Voichick, Extreme points in spaces of analytic functions (to appear).

4. H. Royden, The boundary values of analytic and harmonic functions, Math. Zeitschr. 78 (1962), 1-24.

5. M. Tsuji, Potential theory in modern function theory, Tokyo, 1959.

Received December 18, 1967. 



\section{PACIFIC JOURNAL OF MATHEMATICS}

\section{EDITORS}

H. ROYDEN

Stanford University

Stanford, California

R. R Phelps

University of Washington

Seattle, Washington 98105
J. DUGUNDJI

Department of Mathematics

University of Southern California

Los Angeles, California 90007

RICHARD ARENS

University of California

Los Angeles, California 90024

\section{ASSOCIATE EDITORS}
E. F. BECKENBACH
B. H. NeumanN
F. WOLF
K. YosidA

\section{SUPPORTING INSTITUTIONS}

UNIVERSITY OF BRITISH COLUMBIA

CALIFORNIA INSTITUTE OF TECHNOLOGY

UNIVERSITY OF CALIFORNIA

MONTANA STATE UNIVERSITY

UNIVERSITY OF NEVADA

NEW MEXICO STATE UNIVERSITY

OREGON STATE UNIVERSITY

UNIVERSITY OF OREGON

OSAKA UNIVERSITY

UNIVERSITY OF SOUTHERN CALIFORNIA
STANFORD UNIVERSITY

UNIVERSITY OF TOKYO

UNIVERSITY OF UTAH

WASHINGTON STATE UNIVERSITY

UNIVERSITY OF WASHINGTON

AMERICAN MATHEMATICAL SOCIETY CHEVRON RESEARCH CORPORATION TRW SYSTEMS

NAVAL WEAPONS CENTER 


\section{Pacific Journal of Mathematics

Vol. 28, No. $1 \quad$ March, 1969

Patrick Robert Ahern, On the geometry of the unit ball in the space of real annihilating measures .............................. 1

Kirby Alan Baker, Equational classes of modular lattices ............. 9

E. F. Beckenbach and Gerald Andrew Hutchison, Meromorphic minimal surfaces ......................................... 17

Tae Ho Choe, Intrinsic topologies in a topological lattice ..............

John Bligh Conway, A theorem on sequential convergence of measures and

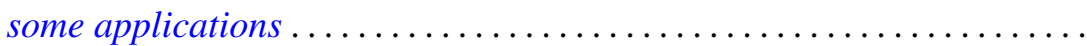

Roger Cuppens, On the decomposition of infinitely divisible probability laws without normal factor.

Lynn Harry Erbe, Nonoscillatory solutions of second order nonlinear

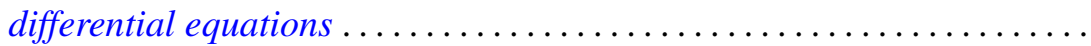

Burton I. Fein, The Schur index for projective representations of finite

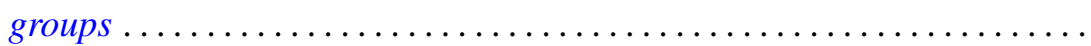

Stanley P. Gudder, A note on proposition observables............... 101

Kenneth Kapp, On Croisot's theory of decompositions ............... 105

Robert P. Kaufman, Gap series and an example to Malliavin's theorem . . . 117

E. J. McShane, Robert Breckenridge Warfield, Jr. and V. M. Warfield,

Invariant extensions of linear functionals, with applications to measures and stochastic processes ................

Marvin Victor Mielke, Rearrangement of spherical modifications ...

Akio Osada, On unicity of capacity functions ..............

Donald Steven Passman, Some 5/2 transitive permutation groups ...

Harold L. Peterson, Jr., Regular and irregular measures on groups and dyadic spaces...

Habib Salehi, On interpolation of $q$-variate stationary stochastic processes...

Michael Samuel Skaff, Vector valued Orlicz spaces generalized

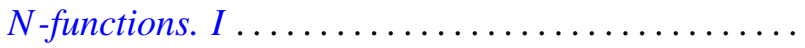

A. J. Ward, On $H$-equivalence of uniformities. II...........

Thomas Paul Whaley, Algebras satisfying the descending chain condition for subalgebras...

G. K. White, On subgroups of fixed index

Martin Michael Zuckerman, A unifying condition for implications among the axioms of choice for finite sets ................. 